\title{
Silymarin-loaded solid nanoparticles provide excellent hepatic protection: physicochemical characterization and in vivo evaluation
}

This article was published in the following Dove Press journal:

International Journal of Nanomedicine

27 August 2013

Number of times this article has been viewed

\author{
Kwan Yeol Yangl,* \\ Du Hyeong Hwangl,* \\ Abid Mehmood Yousaf ${ }^{2}$ \\ Dong Wuk Kim² \\ Young-Jun Shin ${ }^{2}$ \\ Ok-Nam Bae ${ }^{2}$ \\ Yong-II Kim' \\ Jong Oh Kim' \\ Chul Soon Yong' \\ Han-Gon Choi ${ }^{2}$ \\ 'College of Pharmacy, Yeungnam \\ University, Dae-Dong, Gyongsan, \\ ${ }^{2}$ College of Pharmacy and Institute \\ of Pharmaceutical Science and \\ Technology, Hanyang University, \\ Sangnok-gu, Ansan, South Korea \\ "These authors contributed equally \\ to this work
}

Background: The purpose of this study was to develop a novel silymarin-loaded solid nanoparticle system with enhanced oral bioavailability and an ability to provide excellent hepatic protection for poorly water-soluble drugs using Shirasu porous glass (SPG) membrane emulsification and a spray-drying technique.

Methods: A silymarin-loaded liquid nanoemulsion was formulated by applying the SPG membrane emulsification technique. This was further converted into solid state nanosized particles by the spray-drying technique. The physicochemical characteristics of these nanoparticles were determined by scanning electron microscopy, differential scanning calorimetry, and powder $\mathrm{X}$-ray diffraction. Their dissolution, bioavailability, and hepatoprotective activity in rats were assessed by comparison with a commercially available silymarin-loaded product.

Results: Formulation of a silymarin-loaded nanoemulsion, comprising silymarin, castor oil, polyvinylpyrrolidone, Transcutol HP, Tween 80 , and water at a weight ratio of 5/3/3/1.25/1.25/100 was accomplished using an SPG membrane emulsification technique at an agitator speed of $700 \mathrm{rpm}$, a feed pressure of $15 \mathrm{kPa}$, and a continuous phase temperature of $25^{\circ} \mathrm{C}$. This resulted in generation of comparatively uniform emulsion globules with a narrow size distribution. Moreover, the silymarin-loaded solid nanoparticles, containing silymarin/castor oil/polyvinylpyrrolidone/Transcutol HP/Tween 80 at a weight ratio of 5/3/3/1.25/1.25, improved about 1,300 -fold drug solubility and retained a mean size of about $210 \mathrm{~nm}$. Silymarin was located in unaltered crystalline form in the nanoparticles. The drug dissolved rapidly from the nanoparticles, reaching nearly $80 \%$ within 15 minutes, indicating three-fold better dissolution than that of the commercial product. Further, the nanoparticles showed a considerably shorter time to peak concentration, a greater area under the concentration-time curve, and a higher maximum concentration of silymarin compared with the commercial product $(P<0.05)$. In particular, the area under the concentration-time curve of the drug provided by the nanoparticles was approximately 1.3-fold greater than that of the commercial product. In addition, the silymarin-loaded nanoparticles significantly reduced carbon tetrachloride-induced hepatotoxicity, indicating improved bioactivity compared with silymarin powder and the commercial product.

Conclusion: Silymarin-loaded nanoparticles developed using SPG membrane emulsification and spray-drying techniques could be a useful system for delivery of poorly water-soluble silymarin while affording excellent hepatic protection.

Keywords: silymarin, nanoparticle, hepatoprotective activity, Shirasu porous glass membrane, enhanced oral bioavailability

\section{Introduction}

Silymarin is a purified mixture of four isomeric flavonoids extracted from the seeds and fruit of the milk thistle plant, Carduus marianus (L.) Gaertn. These isomeric 
flavonolignans include silyb, isosilybin, silychristin, and silydianin. Silybin is the chief active constituent amongst them. ${ }^{1-3}$ Silymarin has been used to treat liver disease and for the supportive treatment of chronic active hepatitis and hepatic cirrhosis. ${ }^{4,5}$ However, the bioavailability of silymarin is quite low due to its poor aqueous solubility. ${ }^{6-8}$ Pharmacokinetic investigations have demonstrated that only $23 \%-47 \%$ of silymarin reaches the systemic circulation after oral administration. ${ }^{9-11}$ Various techniques, such as complexation of silymarin with phosphatidylcholine, ${ }^{12}$ lecithin, ${ }^{13}$ or cyclodextrin clathrate ${ }^{14}$ and incorporation of silymarin into a solid dispersion ${ }^{15}$ have been used to improve the aqueous solubility of silymarin. Moreover, a commercial product (Legalon $^{\circledR}$, Bukwang Pharmaceutical Company, Seoul, South Korea), which is administered orally twice daily as a hard capsule containing $70 \mathrm{mg}$ silymarin, has been launched on the market.

In this study, with the intention of developing a new silymarin-loaded solid nanoparticle system with enhanced oral bioavailability of the hydrophobic drug, a silymarinloaded nanoemulsion was formulated using a Shirasu porous glass (SPG) membrane emulsification technique and then spray-dried to obtain solid state nanoparticles. Scanning electron microscopy, differential scanning calorimetry (DSC), and powder X-ray diffraction techniques were used to explore the physicochemical properties of the nanoparticles. Their dissolution, bioavailability in rats, and hepatoprotective activity in carbon tetrachloride $\left(\mathrm{CCl}_{4}\right)$-treated rats were assessed in comparison with the commercial product.

Although liquid nanoemulsions play an important role in improving oral drug bioavailability and reducing various side effects, few emulsion preparations are currently in use when compared with other oral dosage formulations. Their less frequent use is because of their poor physicochemical stability and compliance issues. Emulsions are notorious for their physical instability. One way to circumvent this problem and render emulsions more attractive for use could be to transform them into dry powdered nanoemulsions. First described in the early 1960s, a dry powdered emulsion, a type of nanoparticle, is a dispersion of an immiscible oil phase within a solid phase, and is achieved by elimination of the aqueous phase of a liquid emulsion by spray-drying, ${ }^{16}$ lyophilization, ${ }^{17}$ and later solvent evaporation. ${ }^{18,19}$ These nanoparticles are lipid-based powder formulations from which an oil/water $(\mathrm{o} / \mathrm{w})$ emulsion can be reconstituted in vivo or in vitro. Like o/w emulsions, these nanoparticles represent a potential oral dosage form for lipophilic and poorly water-soluble drug entities.
In this study, the SPG membrane emulsification technique was used because of its uniform-sized pores and the wide range of mean pore diameters $(0.05-50 \mu \mathrm{m})$ available. In addition, it is capable of generating narrow droplet size distributions by expending low energy. Since the development of uniform-sized kerosene-in-water and water-in-kerosene emulsions, this technique has attracted the attention of many investigators because of its ability to produce narrow droplet size distributions with low energy consumption. ${ }^{18}$ SPG membranes are potentially amenable to membrane emulsification because they have uniform-sized pores and a wide range of available mean pore diameters. ${ }^{20}$ When nanoparticles are developed conventionally, irregularly sized nanoparticles are produced due to lack of inclusion of an SPG membrane emulsification process. In this study, SPG membrane emulsification yielded uniform emulsion droplets with a narrow size distribution via spray-drying. ${ }^{21}$

\section{Materials and methods}

Silymarin was supplied by Kolon Life Science Company (Kwacheon, South Korea). Transcutol HP, polysorbate 80 (Tween 80), castor oil, and polyvinylpyrrolidone (PVP K30) were obtained from Gattefosse (Saint-Priest Cedex, France), Duksan Chemical Company (Ansan, South Korea), SigmaAldrich (St Louis, MO, USA) and Shin-Etsu Co (Tokyo, Japan), respectively. The commercially available silymarin product (Legalon ${ }^{\circledR}$, in capsule form) was sourced from Bukwang Pharmaceutical Company (Seoul, South Korea). All other chemicals were of reagent grade and used without further purification.

\section{Animals}

All animal care and procedures were conducted in accordance with the Guiding Principles in the Use of Animals in Toxicology, as adopted in 1989, revised in 1999, and amended in 2008 by the Society of Toxicology. ${ }^{22}$ The protocols used for the pharmacokinetic studies were also sanctioned by the Institute of Laboratory Animal Resources at Yeungnam University. Sixteen male Sprague Dawley rats (aged 7-9 weeks, weighing 260-300 g) were purchased from Charles River Company (Seoul, Korea). Further in vivo animal experiments for hepatotoxicity were performed using 25 male Sprague Dawley rats weighing 190-210 g (Charles River Company) and performed in accordance with the National Institutes of Health Policy and Animal Welfare Act under the approval of the Institutional Animal Care and Use Committee at Hanyang University. The rats were housed at a temperature of $20^{\circ} \mathrm{C}$ to $23^{\circ} \mathrm{C}$ with a relative humidity of 
$50 \% \pm 5 \%$, and fasted for 24-36 hours prior to the experiments, but were allowed free access to water.

\section{Solubility}

An excess of silymarin powder (about $100 \mathrm{mg}$ ) was transferred to $1 \mathrm{~mL}$ of surfactant, oil, or aqueous solution containing $1 \%$ hydrophilic polymer in a conical tube. The tubes were agitated in a water bath at $25^{\circ} \mathrm{C}$ for 7 days, centrifuged at $3,000 \mathrm{~g}$ for 10 minutes using a $5415 \mathrm{C}$ centrifuge (Eppendorf, Hauppauge, NY, USA) and filtered through a membrane filter $(0.45 \mu \mathrm{m})$. The silymarin in the filtrate was assayed using a high-pressure liquid chromatography (HPLC) system (Hitachi, Tokyo, Japan) equipped with Ezchrom elite (version 318a) computer software, a Hitachi L-2130 pump, and a Hitachi L-2400 ultraviolet-visible detector. An Inertsil ODS-2 C18 column (GL Science, Tokyo, Japan; $0.5 \mu \mathrm{m}$, $15 \mathrm{~cm} \times 0.46 \mathrm{~cm}$ internal diameter) was used in this analysis. The mobile phase A consisted of a mixture of methanol, acetic acid, and phosphate buffer ( $\mathrm{pH} 3.0,34 / 6 / 60, \mathrm{v} / \mathrm{v} / \mathrm{v})$, and the mobile phase $\mathrm{B}$, acetonitrile, was eluted as follows: 0 minutes (98:2), 0.1 minute (80:20), 12 minutes (98:2) and 22 minutes (98:2). The eluent ( $1 \mathrm{~mL}$ per minute) was monitored at $285 \mathrm{~nm}$ for detection of silymarin. The interday and intraday variance of this HPLC method was within the acceptable range $\left(R^{2}=0.999\right)$.

\section{Pseudoternary phase diagram}

The existence of emulsion fields forming emulsions on agitation was identified from ternary phase diagrams of systems comprising oil-surfactant-water. The influence of surfactant (Transcutol HP, Tween 80, or a mixture of these two surfactants at a 1:1 w/w ratio) and water on the pseudoternary phase diagram was carefully recorded at room temperature. Subsequently, the oil phase (castor oil) was added in 5\% increments to each clear mixture of water and surfactant. This mixture was vortexed for 5 minutes and placed in a water bath at $25^{\circ} \mathrm{C}$ for 30 minutes. The mixture was then visually inspected for formation of an emulsion. The points from uniform turbid solution to separation were considered as the emulsion-forming and nonemulsion-forming regions, respectively.

\section{Experimental setup and procedure}

An emulsification kit comprising an MPG module (microporous glass, $\mathrm{SPG}^{\circledR}$; Kiyomoto Iron Works Company, Miyazaki, Japan) and a hydrophilic SPG membrane (outer diameter $10 \mathrm{~mm}$, thickness $0.75 \mathrm{~mm}$, pore size $2.5 \mu \mathrm{m}$ ) was used. ${ }^{21,23,24}$ Ten grams of dispersed (oil) phase was retained in an air-tight vessel connected to a nitrogen gas inlet connected in turn to a pressure gauge (PG-200-163GP-S, Copal Electronics, Tokyo, Japan). The continuous phase, $100 \mathrm{~g}$ of water containing $2.5 \mathrm{~g}$ surfactant and $3 \mathrm{~g}$ polyvinylpyrrolidone, was stirred gently with a magnetic bar $(3 \mathrm{~cm}$ long) in a beaker to avoid creaming of droplets. By exerting an appropriate pressure of nitrogen gas, the oil phase $(3 \mathrm{~g}$ castor oil) was infused through the uniform pores of the membrane into the aqueous phase to produce globules. These dispersed globules were stabilized by polyvinylpyrrolidone dissolved in aqueous phase. The experiments were conducted over a wide range of agitator speeds (300-700 rpm), feed pressures $(15-35 \mathrm{kPa})$, and continuous phase temperatures $\left(25^{\circ} \mathrm{C}-45^{\circ} \mathrm{C}\right)$.

\section{Preparation of silymarin-loaded solid nanoparticles}

A Büchi 190 nozzle-type mini spray dryer (Flawil, Switzerland) was used to form the silymarin-loaded nanoparticles. The silymarin-loaded nanoemulsion was carried to the spraying nozzle $(0.7 \mathrm{~mm}$ diameter $)$ at a flow rate of $5 \mathrm{~mL}$ per minute by a peristaltic pump, and spray-dried at an inlet temperature of $120^{\circ} \mathrm{C}$ and an outlet temperature of $75^{\circ} \mathrm{C}-80^{\circ} \mathrm{C}$. The pressure of the sprayed air was $4 \mathrm{~kg} / \mathrm{cm}^{2}$. The flow rate of drying air was maintained at an aspirator setting of 10 , indicating that the pressure of the aspirator filter vessel was -25 mbar. The sprayed products were delivered in the direction of air flow.

\section{Mean droplet size and distribution of silymarin-loaded nanoparticles}

The droplet size distribution and zeta potential of the silymarin nanoemulsion and solid nanoparticles were investigated using a Zetasizer NanoZS light dispersing particle size analyzer (Malvern Instruments Ltd, Malvern, UK) with noninvasive back scatter $\left(\mathrm{NIBS}^{\circledR}\right)$ technology, which permitted detection of globules within the range of 0.6-6,000 nm. Acquisition of data on particle size distribution was done using the DTS (nano) software (version 5.0) supplied with the device. The z-average diameter, also referred to as the harmonic intensity-weighted average hydrodynamic diameter, of the emulsions was calculated from cumulated analysis applying Automeasure software (Malvern Instruments Ltd). For investigation of the silymarin-loaded nanoparticle size, reconstitution was achieved prior to measurement as follows: the nanoparticles $(6 \mathrm{mg})$ were vortexed with $10 \mathrm{~mL}$ of distilled water in a conical tube for 10 seconds, and subsequently incubated for 30 minutes at $25^{\circ} \mathrm{C}$. 


\section{Morphologic features of silymarin- loaded nanoparticles}

The morphologic manifestations associated with the shape and surface of the silymarin powder and silymarin-loaded solid nanoparticles were studied using a scanning electron microscope (S-4100, Hitachi) equipped with an image analysis system (ImageInside version 2.32). The powders were stuck onto a brass specimen club using double-sided adhesive tape and made electrically conductive by coating in a vacuum (6 Pa) with platinum (6 nm per minute) using a Hitachi Ion Sputter (E-1030) for 300 seconds at $15 \mathrm{~mA}$.

\section{Thermal and structural characterization of silymarin-loaded nanoparticles}

The thermal aspects of silymarin powder, polyvinylpyrrolidone, the physical mixture, and silymarin-loaded solid nanoparticles were evaluated using a differential scanning calorimeter (DSC2010, TA Instruments, New Castle, DE, USA). The physical mixture was constituted by physically mingling silymarin and polyvinylpyrrolidone at a weight ratio of 1:1. Approximately $2 \mathrm{mg}$ of each sample was enclosed in a sealed aluminum pan prior to heating under nitrogen flow $(25 \mathrm{~mL}$ per minute) at a heating rate of $10^{\circ} \mathrm{C}$ per minute in the range of $10^{\circ} \mathrm{C}-180^{\circ} \mathrm{C}$. The crystallinity of the drug in the nanoparticles was measured by powder X-ray diffraction (MPD for bulk, PANalytical, Almelo, The Netherlands) performed at room temperature using monochromatic $\mathrm{Cu}-\mathrm{K} \alpha$ radiation $(\lambda=1.54178 \AA)$ at $30 \mathrm{~mA}$ current and $40 \mathrm{kV}$ voltage in the region of $10^{\circ} \leq 2 \theta \leq 40^{\circ}$ with an angular increment of $0.02^{\circ}$ per second.

\section{Dissolution}

Dissolution studies were performed using a basket method (USP XXI, apparatus I). The drug powder, silymarin-loaded nanoparticles, and the commercial product, equivalent to $140 \mathrm{mg}$ of silymarin, were introduced into the basket and placed in the dissolution tester (Shinseang Instrument Company, Seoul, South Korea). This apparatus was fitted with an outer waterbath in order to regulate temperature and sink conditions. The dissolution test was conducted at $36.5^{\circ} \mathrm{C}$ using the rotating basket method at 100 rpm in $900 \mathrm{~mL}$ of water as the dissolution medium. At scheduled intervals, $2 \mathrm{~mL}$ of the dissolution medium was withdrawn and filtered through a membrane filter $(0.45 \mu \mathrm{m}$, nylon syringe filter). The concentration of silymarin in the filtrate $(50 \mu \mathrm{L})$ was analyzed by HPLC, as mentioned above.

\section{Pharmacokinetics}

Twelve rats, divided into two groups, were orally administered the commercial product or nanoparticles, each at a silymarin dose of $10 \mathrm{mg} / \mathrm{kg}$. Each rat, anesthetized in an ether-saturated chamber, was fastened onto a surgical board in the supine position with a thread. A polyethylene tube was introduced into the right femoral artery of the rat. The nanoparticles and commercial product, enclosed in small hard gelatin capsules (\#9, Suheung Capsule Company, Seoul, Korea), were orally administered to the rats in each group. Afterwards, $0.2 \mathrm{~mL}$ of blood was sampled from the right femoral artery at preset time intervals and centrifuged at 3,000 $\mathrm{g}$ for 10 minutes using a $5415 \mathrm{C}$ centrifuge (Eppendorf).

\section{Blood sample analysis}

Plasma $(150 \mu \mathrm{L})$ was poured into $400 \mu \mathrm{L}$ of $\mathrm{pH} 5.0$ acetate buffer solution, $50 \mu \mathrm{L}$ of internal standard working solution (ethylparaben $20 \mu \mathrm{g} / \mathrm{mL}$ of methanol), and $50 \mu \mathrm{L}$ of purified enzyme solution. The purified enzyme solution comprised 13.48 units of $\beta$-glucuronidase and 4.5 units of arylsulfatase in $0.5 \mathrm{M}$ acetate buffer ( $\mathrm{pH}$ 5.0). This resulting solution was kept at $37^{\circ} \mathrm{C}$ for 4 hours to promote cleavage of glucuronides and sulfates of silybin. The solution was then cooled to room temperature, $1.5 \mathrm{~mL}$ of acetonitrile was introduced, and the solution was shaken vigorously for 15 minutes. After centrifuging at 2,000 rpm for 10 minutes, the supernatant layer was transferred into a microtube and evaporated. The residue was reconstituted with $100 \mu \mathrm{L}$ of the mobile phase, vortexed for one minute, and centrifuged at 10,000 rpm for 5 minutes. Next, $50 \mu \mathrm{L}$ of the supernatant layer was assayed by HPLC (Hitachi) equipped with an Inertsil ODS-2 C18 column and an ultraviolet detector (Model L-7450). The mobile phase was composed of methanol and $\mathrm{pH} 3.0$ phosphate-buffered solution $(45 / 55, \mathrm{v} / \mathrm{v})$. The eluent was monitored at $285 \mathrm{~nm}$ with a flow rate of $1.0 \mathrm{~mL}$ per minute for drug detection.

\section{Hepatoprotective activity}

Induction of hepatotoxicity

Hepatotoxicity was induced by $\mathrm{CCl}_{4}$ as previously described. ${ }^{25,26}$ Twenty-five rats were divided into five groups. Rats in one group were not given the treatment, and those in the second group were administered the treatment with $0.5 \%$ sodium carboxymethylcellulose as a vehicle. To evaluate the protective activities of silymarin powder, commercial product, or nanoparticles, a dose of $50 \mathrm{mg} / \mathrm{kg}$ was administered orally to each rat in the other groups on days 1 and 2. In all groups, hepatotoxicity was induced by treatment with $\mathrm{CCl}_{4}(50 \%$ in corn oil, $0.5 \mathrm{~mL} / \mathrm{kg}$ body weight, orally) on day 3 . All animals were fasted for 16 hours prior to administration of $\mathrm{CCl}_{4}$. One hour after administration of $\mathrm{CCl}_{4}$, the rats were allowed access to food and water ad libitum. Each formulation of silymarin 
was administered to $\mathrm{CCl}_{4}$-exposed rats on day 4 , and the rats were sacrificed on day 5 .

\section{Evaluation of hepatoprotection}

$\mathrm{CCl}_{4}$-induced hepatotoxicity was determined by serum aspartate aminotransferase levels and histopathologic examination. Blood samples were obtained from the abdominal aorta, and the serum was isolated by centrifugation at $12,000 \mathrm{~g}$ for 5 minutes. Serum aspartate aminotransferase levels were estimated by colorimetry, measuring the amount of oxaloacetate produced. For histopathologic evaluation, the liver was rapidly harvested and fixed in 4\% paraformaldehyde. After staining with hematoxylin-eosin, the liver sections were visually examined.

\section{Results and discussion Solubility}

An emulsion system consisting of oil, surfactants, cosurfactants, and drugs should possess good solvent properties to permit presentation of the drug in solution. The solubility of silymarin in water is about $6.2 \mu \mathrm{g} / \mathrm{mL}$, indicating that this drug is poorly water-soluble. ${ }^{11}$ Amongst the oils and hydrophilic polymers tested (data not shown), castor oil and polyvinylpyrrolidone enabled the highest solubility of silymarin, so were nominated as an oil phase and stabilizer, respectively. The selection of surfactants is significant for generation of uniformly-sized emulsion droplets via SPG membrane emulsification. The drug was more soluble in all of the surfactants compared with its aqueous solubility. The hydrophilic functional groups of the surfactants selected must not carry the opposite charge to that of the membrane surface in order to avoid electrostatic attractions with the membrane surface, which has a negative zeta potential within the $\mathrm{pH}$ range of 2-8, due to the dissociation of silanol groups on the membrane surface. ${ }^{27,28}$ Accordingly, cationic surfactants must be avoided when using SPG membranes. Silymarin showed the highest solubility of about $170 \mathrm{mg} / \mathrm{mL}$ in Transcutol HP, because Transcutol HP was considered a powerful solubilizing agent used in numerous dosage forms because of its ability to solubilize several drug substances. ${ }^{29}$ Further, Tween 80 offered higher drug solubility than other the surfactants. Consequently, Transcutol HP and Tween 80, both nonionic surfactants, were chosen as surfactants.

\section{Pseudoternary phase diagrams}

Construction of a phase diagram facilitates identification of the range of concentrations of components which constitute nanoemulsions. Phase diagrams were constructed to appraise the individual component ratio responsible for formation of an o/w nanoemulsion consisting of castor oil (oil phase), a surfactant mixture of Transcutol HP/Tween 80 (1:1), and water (Figure 1). The larger nanoemulsion region was attained in the system in which the surfactant mixture was used compared with Transcutol HP or Tween 80 alone. Furthermore, the area in which an o/w nanoemulsion formed altered with the surfactant. The formulation composed of castor oil, polyvinylpyrrolidone, Transcutol HP, Tween 80, and water at the weight ratio of 3/3/1.25/1.25/100 was adopted as the basic component, because use of the surfactant mixture and the maximum amount of water could enable silymarin to be solubilized in the nanoemulsion system. ${ }^{21,23,24}$ Likewise, because $5 \mathrm{~g}$ of silymarin is completely soluble in this emulsion system, the formulation composed of silymarin, castor oil, polyvinylpyrrolidone, Transcutol HP, Tween 80 , and water at the weight ratio of 5/3/3/1.25/1.25/100 was chosen as a silymarin-loaded nanoemulsion for further study.

\section{Influence of process parameters on mean droplet size and distribution} Effect of agitator speed

The impact of agitator speed over a range of 300-700 rpm on the z-average diameter of nanoemulsion droplets of

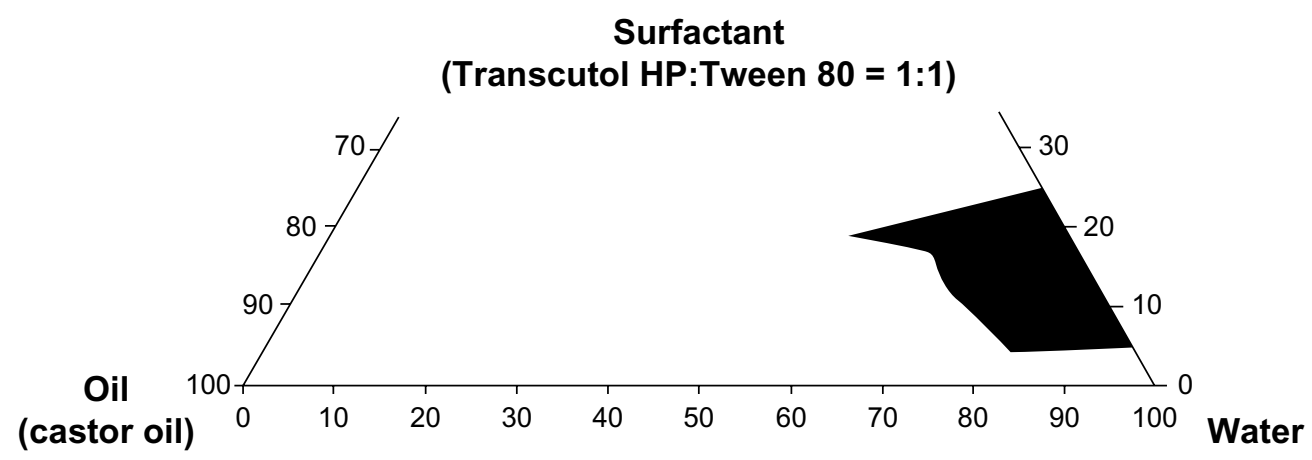

Figure I Pseudoternary phase diagrams plotted with castor oil, Transcutol HP/Tween 80 ( I:I, volume ratio), and water. 
Z-average diameter for the $2.5 \mu \mathrm{m}$ SPG membrane is shown in Figure 2A. The z-average diameters of the nanoemulsions prepared with the membrane were considerably diminished compared with those prepared by mere stirring without the membrane. The $\mathrm{z}$-average diameter decreased sharply as the agitator speed was increased to $700 \mathrm{rpm}$. Our results suggest that an agitator speed of $700 \mathrm{rpm}$ is optimal, given that it resulted in emulsion droplets with a relatively smaller z-average diameter. In prior investigations, the mean particle diameter was reduced with an acceleration in stirring rate because the nanoemulsion droplets produced by the emulsification technique were more prone to coalescence compared with other droplets having surfaces stabilized by the surfactants and counterdiffusion of both solvents was completed before the droplets sufficiently dispersed in the poor solvent, the upsurge in stirring rate improved droplet distribution, prevented droplet coalescence, and caused in a smaller apparent particle size of nanoemulsion. ${ }^{30}$

\section{Effect of feed pressure of dispersion phase}

Another parameter with an intense impact on droplet size distribution is feed pressure, which can control the flux rate of the dispersed phase across the membrane channel and detachment of the droplets. A diminution in feed pressure resulted in formation of nanoemulsion droplets with a smaller z-average diameter (Figure 2B). As the dispersed-phase transmembrane pressure increased from $15 \mathrm{kPa}$ to $35 \mathrm{kPa}$, the droplet size increased from $156.7 \mathrm{~nm}$ to $496.7 \mathrm{~nm}$; this substantial enlargement in mean droplet size was because of droplet growth and coalescence at the membrane surface within the period of decreasing interfacial tension. Similar outcomes were also seen in previous studies. ${ }^{31-33}$ As shown in Figure 2B, increasing transmembrane pressure also prompted an increased size distribution, as indicated by a larger standard deviation. Thus, an optimized transmembrane pressure of $15 \mathrm{kPa}$ was obtained.

\section{Effect of continuous phase temperature}

Temperature can be an imperative factor in emulsification, affecting the viscosity of both the dispersed and continuous phases (Figure 2C). Further, it can affect the nature and solubility of the emulsifier as a consequence of the phase inversion temperature. ${ }^{34}$ In this study, castor oil was used as the oil phase. The membrane nanoemulsion formulated with a continuous phase temperature of $25^{\circ} \mathrm{C}$ had the smallest mean droplet size with a narrow size distribution; however, as the continuous phase temperature was elevated from $30^{\circ} \mathrm{C}$ to $45^{\circ} \mathrm{C}$, the mean droplet size increased accordingly. In particular, a remarkable increase in mean droplet size was found when the temperature was increased from $40^{\circ} \mathrm{C}$ to $45^{\circ} \mathrm{C}$. Increasing temperature will result in an increase in emulsion droplet size and polydispersity owing to coarsening by Ostwald ripening and/or coalescence. ${ }^{35}$ Accordingly, an optimized continuous phase temperature of $25^{\circ} \mathrm{C}$ was achieved.

On the basis of these conclusions, the silymarin-loaded nanoemulsion to be used for further study was accomplished with the formulation consisting of silymarin, castor oil, polyvinylpyrrolidone, Transcutol HP, Tween 80 , and water at the weight ratio of 5/3/3/1.25/1.25/100 using an SPG membrane at an agitator speed of $700 \mathrm{rpm}$, a feed pressure of $15 \mathrm{kPa}$, and a continuous phase temperature of $25^{\circ} \mathrm{C}$.
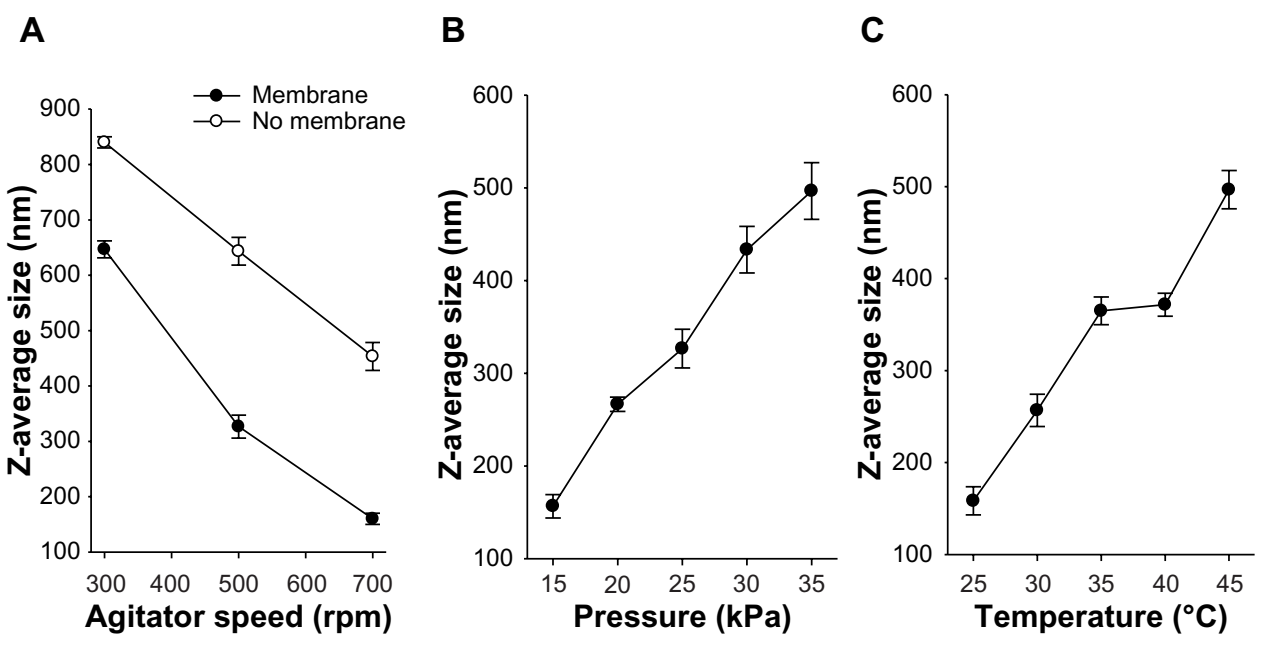

Figure 2 Effect of agitator speed $(\mathbf{A})$, feed pressure $(\mathbf{B})$, and continuous phase temperature $(\mathbf{C})$ on the z-average diameter of the emulsion droplet. Each value represents the mean \pm standard deviation $(n=3)$. 


\section{Silymarin-loaded solid nanoparticles}

The silymarin-loaded solid nanoparticles obtained by spraydrying the silymarin-loaded nanoemulsion were considerably dry and nonsticky. These nanoparticles comprised silymarin/ castor oil/polyvinylpyrrolidone/Transcutol HP/Tween 80 at a weight ratio of 5/3/3/1.25/1.25. The mean sizes of the silymarin-loaded nanoemulsion and nanoparticles were $169.1 \pm 1.5 \mathrm{~nm}$ and $214.3 \pm 1.9 \mathrm{~nm}$, respectively (raw data not shown), showing that the latter had a comparatively larger particle size and broader distribution than the former. Furthermore, the nanoemulsion and nanoparticles had polydispersity values of $0.211 \pm 0.003$ and $0.342 \pm 0.001$, respectively. In the preparation of nanoparticles, solidification by spray-drying did not alter the particle size or size distribution of the particles to a great extent. Nevertheless, the particle size of the reconstituted nanoparticles was well within the nanoscale range. After reconstitution, the nanoparticles resulted in a drug solubility of $8.24 \pm 0.49 \mathrm{mg} / \mathrm{mL}$, which was almost 1,300 -fold better than the solubility of silymarin powder (about $6.2 \mu \mathrm{g} / \mathrm{mL}$ ). ${ }^{11}$

Scanning electron micrographs of the silymarin powder and nanoparticles are shown in Figure 3. The nanoparticles were composed of silymarin/castor oil/polyvinylpyrrolidone/ Transcutol HP/Tween 80 at the weight ratio of 5/3/3/1.25/1.25. Silymarin powder (Figures 3A and 3B) appeared as smoothsurfaced rectangular crystals. However, the nanoparticles (Figures 3C and 3D) had the same external macroscopic morphology, depicting aggregated particles with rather deep dents and similar diameters. Since the particle size of the nanoparticles was reduced to around $210 \mathrm{~nm}$ (as mentioned above) after reconstitution, the enlarged particle size in the
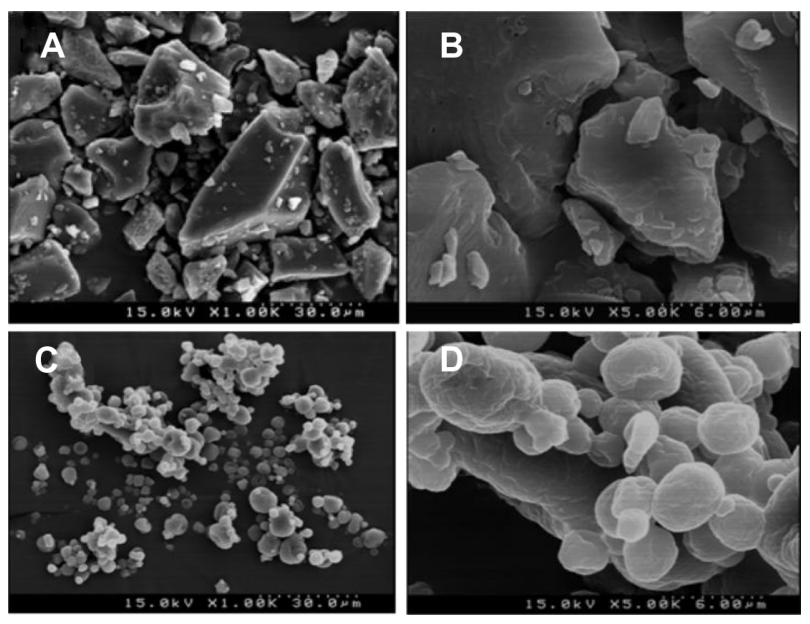

Figure 3 Scanning electron micrographs for (A) silymarin powder $(1,000 \times)$, (B), silymarin powder $(5,000 \times),($ C) nanoparticles $(I, 000 \times)$, and (D) nanoparticles $(5,000 \times)$. solid form was attributed to the attachment of polyvinylpyrrolidone or aggregation. Nevertheless, it conserved the nanoscale properties after reconstitution or redispersion.

The thermal behavior of the drug powder, polyvinylpyrrolidone, the physical mixture, and the nanoparticles is shown in Figure 4A. The DSC thermogram reveals that silymarin generated a broad endothermic peak, indicating that silymarin lacks typical crystallinity which contributes indicative peaks in thermograms of DSC (Figure 4A, A1).$^{36}$ The broad endotherm peak ranging from $70^{\circ} \mathrm{C}$ to $130^{\circ} \mathrm{C}$ for polyvinylpyrrolidone represents loss of water due to the poor hygroscopic nature of polyvinylpyrrolidone polymers (Figure 4A, B1) ${ }^{37}$ Although there were no indicative peaks representing typical crystallinity in silymarin and polyvinylpyrrolidone, the features of the physical mixture (Figure 4A, C1) and nanoparticle curve (Figure 4A, D1) demonstrate the characteristic peaks of polyvinylpyrrolidone and silymarin powder.

A

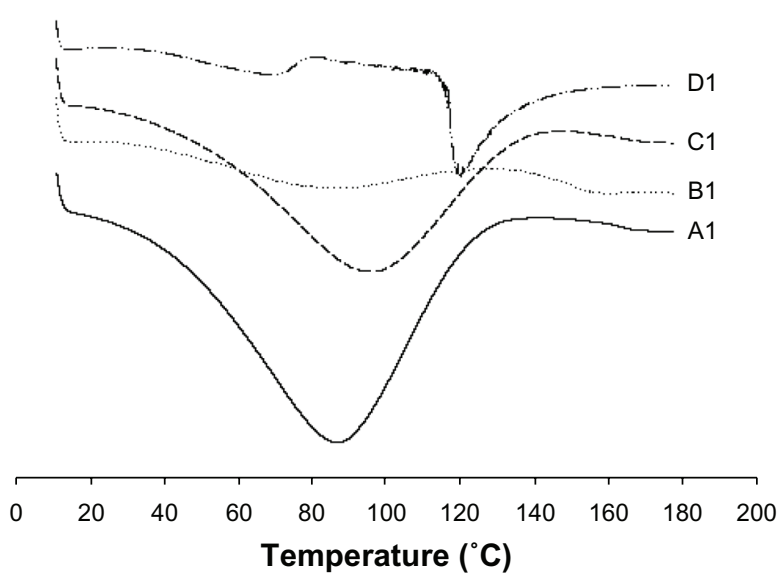

B

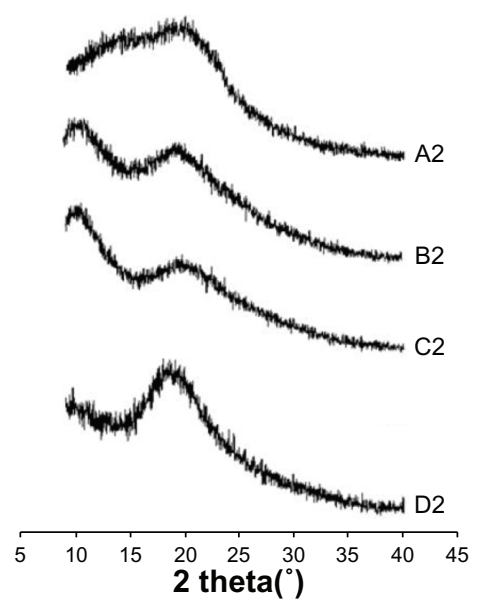

Figure 4 Differential scanning calorimetric thermograms (A) and powder X-ray diffraction (B). (AI and BI), polyvinylpyrrolidone, (A2 and B2), silymarin powder, (CI and C2), physical mixture, and (DI and D2), nanoparticles. 
Hence, in the DSC curve of the nanoparticle and physical mixture, the characteristic peak of silymarin was unaffected, confirming a lack of strong interactions between the drug and carriers for the preparation of nanoparticles. ${ }^{24}$

The powder X-ray diffraction patterns are shown in Figure 4B, and demonstrate that silymarin did not contribute a typical crystalline peak, suggesting that polyvinylpyrrolidone (Figure 4B, B2) and silymarin (Figure 4B, A2) did not possess the typical crystallinity which provided indicative peaks in diagrams of DSC. Moreover, the features of all prominent characteristic crystalline peaks that appeared in drug and polymer cases were detected in the physical mixture (Figure 4B, C2) and nanoparticles (Figure 4B, D2). Therefore, like the DSC results, powder X-ray diffraction also showed that silymarin was present in an unchanged crystalline state in the nanoparticle.

Our physicochemical findings suggest that the improved solubility of poorly water-soluble silymarin in the nanoparticles was not the result of conversion into the amorphous state but due to a reduction in size to nanoscale. ${ }^{38}$

\section{Dissolution}

The dissolution of the drug from the nanoparticles was compared with that of the silymarin powder and silymarin-loaded commercial product. As shown in Figure 5, the nanoparticles enabled a higher dissolution rate of drug compared with silymarin powder and the commercial product. In particular, the drug dissolved rapidly from the nanoparticle and reached about $80 \%$ within 15 minutes. At this point in time, the dissolution rate of silymarin from the nanoparticle was higher

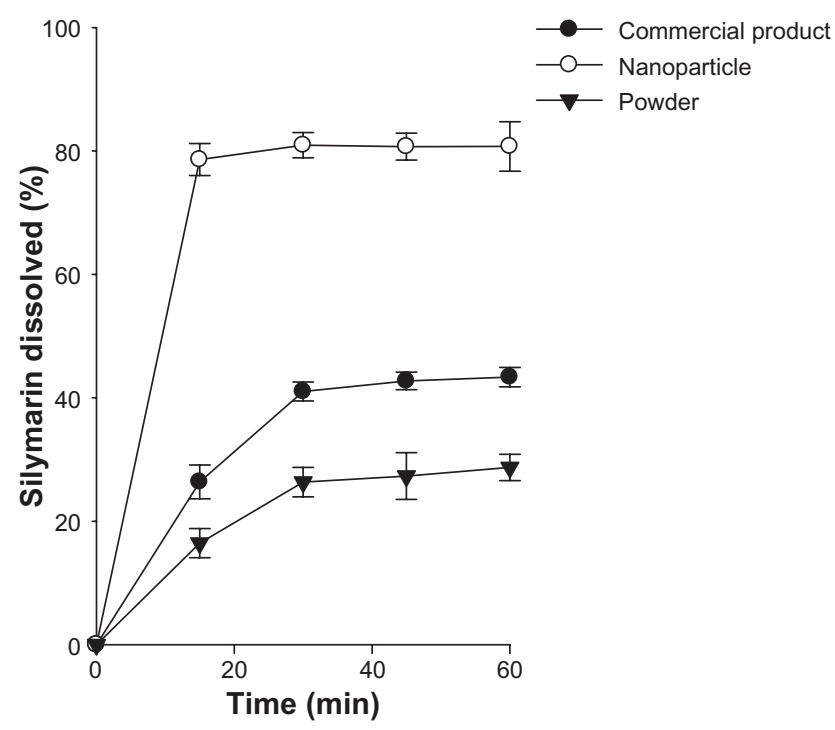

Figure 5 Dissolution profile of the drug from commercial product and nanoparticles in water. Each value represents the mean \pm standard deviation $(n=6)$. than that from the commercial product and silymarin powder (about three-fold and five-fold, respectively). In addition, the amount of drug dissolved from the nanoparticles within 60 minutes was about two-fold and three-fold higher than that from the commercial product and drug powder, respectively. Our results suggest that rapid and complete drug release was achieved by the nanoparticles. Following reconstitution in the dissolution medium, the silymarin in the nanoparticles gave ultralow interfacial tensions and large o/w interfacial areas, leading to incorporation of poorly water-soluble drugs inside the nanosize emulsion droplets. Thus, solid nanoemulsion has an advantage in maintaining high solubilization capacity, which ensures higher dissolution rates in comparison with the crude powder and the commercial product. ${ }^{39,40}$

\section{Pharmacokinetics}

Figure 6 shows the change in mean plasma levels of silymarin in rats following oral administration of the commercial product and nanoparticles at a dose of $10 \mathrm{mg} / \mathrm{kg}$. The total plasma titers of drugs in nanoparticles were higher compared with those of the commercial product. In particular, the initial plasma concentrations of drugs in nanoparticles between

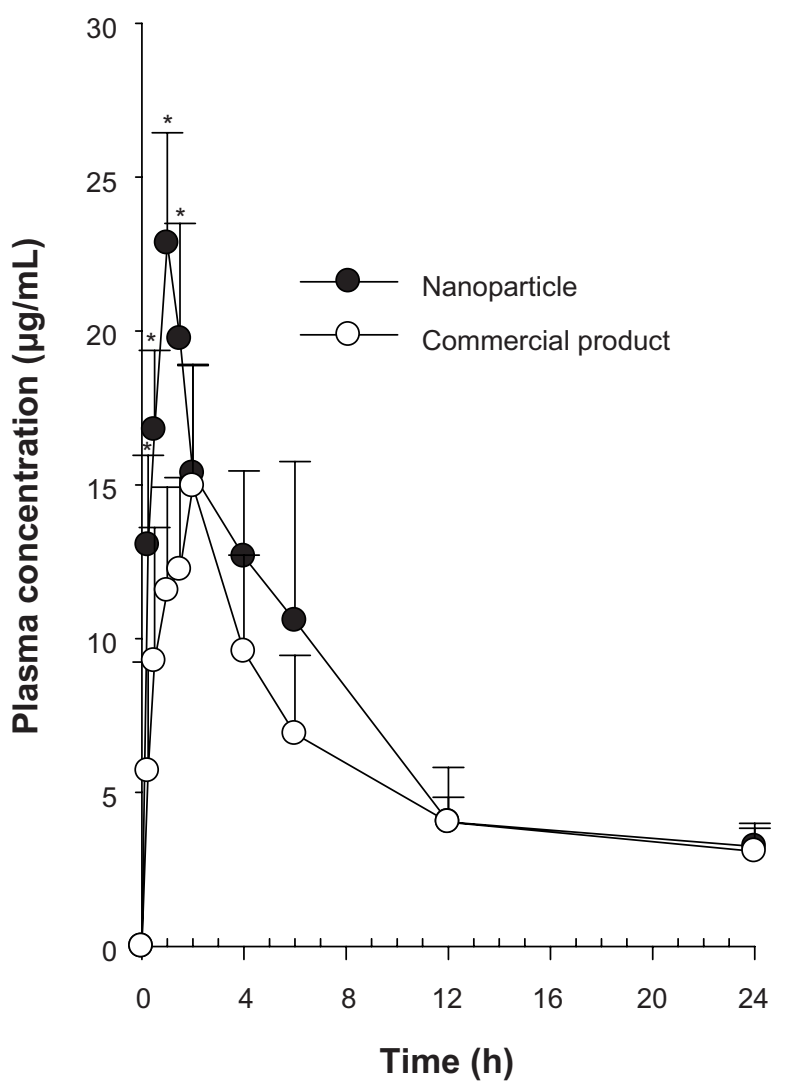

Figure 6 Plasma concentration-time profiles for the drug after oral administration of commercial product and nanoparticles in rats. Each value represents the mean \pm standard deviation $(n=6)$. $* P<0.05$ compared with commercial product. 
Table I Pharmacokinetic parameters

\begin{tabular}{lcc}
\hline Parameters & Nanoparticle & Commercial product \\
\hline $\mathrm{AUC}(\mathrm{h} \cdot \mu \mathrm{g} / \mathrm{mL})$ & $185.34 \pm 25.22^{*}$ & $145.18 \pm 14.49$ \\
$\mathrm{C}_{\max }(\mu \mathrm{g} / \mathrm{mL})$ & $22.07 \pm 2.25^{*}$ & $15.36 \pm 2.25$ \\
$\mathrm{~T}_{\text {max }}$ (hours) & $0.90 \pm 0.26^{*}$ & $\mathrm{I} .82 \pm 0.4 \mathrm{I}$ \\
$\mathrm{t}_{\mathrm{I} / 2}$ (hours) & $2.89 \pm 0.58$ & $2.99 \pm 0.98$ \\
$\mathrm{~K}_{\mathrm{el}}\left(\mathrm{h}^{-1}\right)$ & $0.25 \pm 0.04$ & $0.24 \pm 0.06$ \\
\hline
\end{tabular}

Notes: Each value represents the mean + standard deviation. $(n=6) * P<0.05$ compared with commercial product.

Abbreviations: AUC,area under the drug concentration-time curve from zero to infinity; $\mathrm{C}_{\text {max }}$, maximum plasma concentration of drug; $\mathrm{K}_{\mathrm{e}}$, elimination rate constant; $\mathrm{T}_{\max }$, time taken to reach maximum plasma drug concentration; $\mathrm{t}_{1 / 2}$, elimination half-life.

0.25 and 1.5 hours were impressively higher than those in the commercial product $(P<0.05)$. Our results suggest that the higher initial plasma concentrations of silymarin were as a result of the increased dissolution rate of drug in the nanoparticles. ${ }^{28}$

The pharmacokinetic parameters are shown in Table 1. The nanoparticles provided an impressively greater area under the concentration-time curve and higher peak plasma levels of silymarin than the commercial product (1.27 and 1.44 times, respectively, $P<0.05$ ). Thus, the bioavailability of the drug achieved by the nanoparticles was about 1.3-fold higher than that of the commercial product. On the other hand, the time taken to reach peak drug levels for the nanoparticles was significantly shorter than that for the commercial product $(P<0.05)$. The plasma levels achieved by the nanoparticles increased to $22.85 \pm 3.59 \mu \mathrm{g} / \mathrm{mL}$ at $\mathrm{T}_{\max }$ (time taken to reach peak plasma drug concentration; 1 hour). Our results suggest that a shorter time to peak plasma levels followed by a higher peak plasma concentration was owing to more complete absorption of silymarin in the nanoparticles. Therefore, the enhanced oral bioavailability of silymarin in rats might be associated with marked enhancement in the absorption rate of silymarin as a result of the increased dissolution of drug in the nanoparticles. Conversely, the $\mathrm{K}_{\mathrm{el}}$ (elimination rate constant) and $t_{1 / 2}$ (elimination half-life) values of the drug did not vary considerably between these two groups. ${ }^{21}$ Our results indicate that nanoparticles could be worthwhile for the delivery of drugs in a design that permits rapid absorption in the early phase, resulting in complete and better absorption. ${ }^{23,24}$

\section{Hepatoprotective activity}

To compare the hepatoprotective effects of silymarin-loaded nanoparticles with those of the silymarin powder or the commercial product, the levels of serum transaminase and liver histopathology were evaluated. ${ }^{25,26}{\text { Treatment with } \mathrm{CCl}_{4}}$ significantly elevated the serum aspartate aminotransferase activity, which was significantly reduced by pretreatment with silymarin-loaded nanoparticles (Figure 7A). However, the silymarin powder and commercial product failed to reverse the $\mathrm{CCl}_{4}$-induced elevation of serum aspartate aminotransferase. The serum activity of alanine aminotransferase, another representative biomarker for hepatotoxicity, appeared in a similar pattern to aspartate aminotransferase, confirming the superior bioactivity of silymarin-loaded nanoparticles (data not shown). The protective effects of silymarin-loaded
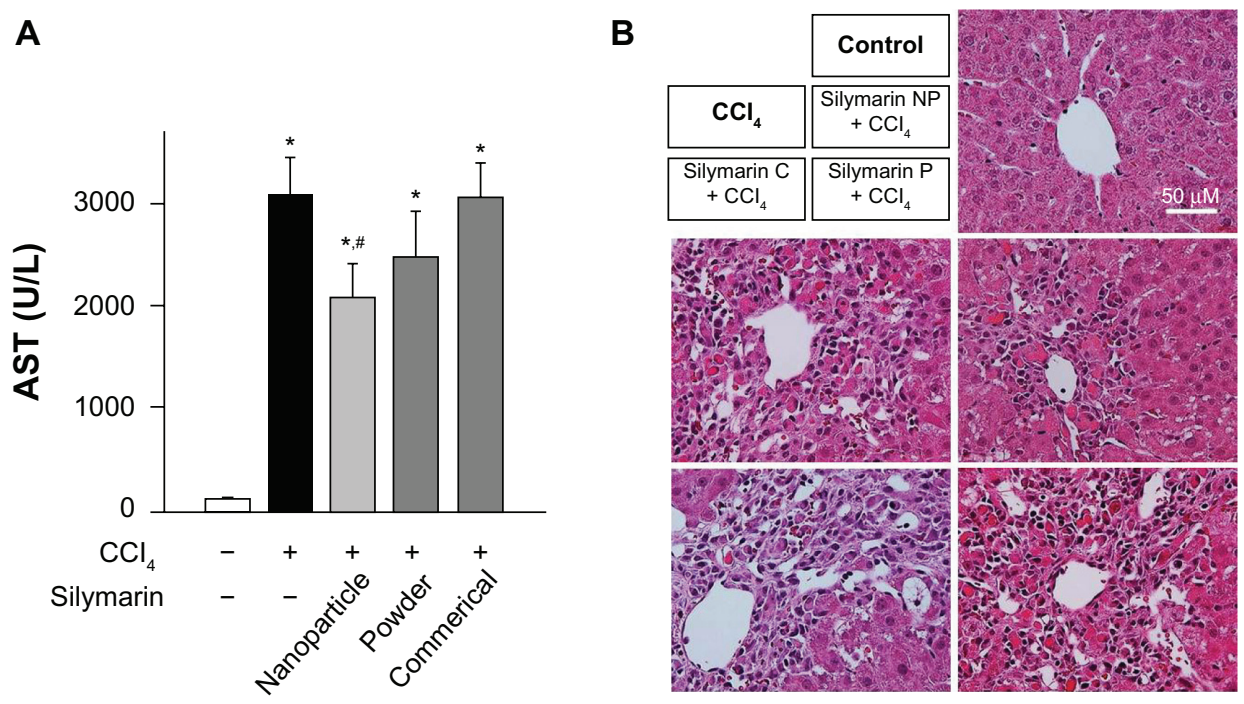

Figure 7 Effect of silymarin-loaded solid nanoparticles on carbon tetrachloride-induced hepatotoxicity. (A) Serum activity of aspartate aminotransferase; each value represents the mean \pm standard deviation. $(n=5)$ and was analyzed by the Student's $t$-test. $* P<0.05$ compared with control group; ${ }^{*} P<0.05$ compared with treated group. (B) Representative photos of hematoxylin-eosin staining of liver sections.

Abbreviations: AST, aspartate aminotransferase; NP, nanoparticles; silymarin C, commercial product of silymarin; silymarin P, silymarin powder; CCl, carbon tetrachloride. 
nanoparticles on $\mathrm{CCl}_{4}$-induced acute liver toxicity were also observed by histopathologic examination. Compared with the healthy livers, which had normal lobular architecture with a central vein and radiating hepatic cords, liver sections from $\mathrm{CCl}_{4}$-exposed rats showed necrosis of hepatocytes, hepatocyte degeneration, and infiltration of inflammatory cells (Figure 7B). While the $\mathrm{CCl}_{4}$-induced destruction of liver architecture was not improved in rats treated with silymarin powder or the commercial product, the liver tissue from rats treated with silymarin nanoparticles showed less severe damage surrounding the central vein, suggesting superior hepatoprotective activity of silymarin nanoparticles over the drug powder or the commercial silymarin product.

\section{Conclusion}

A silymarin-loaded nanoemulsion composed of silymarin, castor oil, polyvinylpyrrolidone, Transcutol HP, Tween 80 , and water at the weight ratio of 5/3/3/1.25/1.25/100 was prepared using an SPG membrane at an agitator speed of $700 \mathrm{rpm}$, a feed pressure of $15 \mathrm{kPa}$, and a continuous phase temperature of $25^{\circ} \mathrm{C}$. It offered comparatively uniform emulsion droplets with a narrow size distribution. Moreover, silymarin-loaded nanoparticles at a weight ratio of silymarin/ castor oil/polyvinylpyrrolidone/Transcutol HP/Tween 80 of 5/3/3/1.25/1.25 were prepared from a silymarin-loaded nanoemulsion using a spray-drying technique. Unlike conventional nanoparticles with a wide size distribution, these nanoparticles had a uniform nanosize of about $210 \mathrm{~nm}$, with a narrow size distribution and enhanced drug solubility of about 1,300-fold. Silymarin was present in the unchanged crystalline state in the nanoparticles. The nanoparticles also took less time to reach peak plasma levels and had a higher area under the concentration-time curve and peak plasma levels of the drug than the commercial product $(P<0.05)$. In particular, the oral bioavailability of the drug from the nanoparticles was about 1.3-fold higher than that obtained with the commercial product. The improved bioactivity of silymarin-loaded nanoparticles was observed in rat acute liver injury models. Hence, these silymarin-loaded nanoparticles prepared using SPG membrane emulsification and spray-drying techniques could be useful to deliver poorly water-soluble silymarin with excellent hepatic protection by enhanced oral bioavailability via nanosized particles. Generally, controlled-release delivery systems cannot be formulated with poorly water-soluble drugs because of their limited aqueous solubility. Thus, for development of a better candidate of silymarin, further study of the controlled-release system will be developed not with poorly water-soluble silymarin powder but with this more soluble silymarin-loaded nanoparticle.

\section{Acknowledgment}

This work was supported by a National Research Foundation of Korea grant funded by the Korea government (N2012R1A2A2A01045658, 2013M4A1035382).

\section{Disclosure}

The authors report no conflicts of interest in this work.

\section{References}

1. Kvasnika F, Biba B, Sevcík R, Voldrich M, Krátká J. Analysis of the active components of silymarin. J Chromatogr A. 2003;990:239-245.

2. Lu C, LuY, Chen J, Zhang W, Wu W. Synchronized and sustained release of multiple components in silymarin from erodible glyceryl monostearate matrix system. Eur J Pharm Biopharm. 2007;66:210-219.

3. Lee JI, Hsu BH, Wu D, Barrett JS. Separation and characterization of silybin, isosilybin, silydianin and silychristin in milk thistle extract by liquid chromatography-electrospray tandem mass spectrometry. J Chromatogr A. 2006;1116:57-68.

4. Fintelmann V. Modern phytotherapy and its uses in gastrointestinal conditions. Planta Med. 1991;57:S48-S52.

5. Flora K, Hahn M, Rosen H, Benner K. Milk thistle (Silybum marianum) for the therapy of liver disease. Am J Gastroenterol. 1998;93: 139-143.

6. Basaga H, Poli G, Tekkaya C, Aras I. Free radical scavenging and anti-oxidative properties of 'silibin' complexes on microsomal lipid peroxidation. Cell Biochem Funct. 1997;15:27-33.

7. Skottová N, Krecman V. Dietary silymarin improves removal of low density lipoproteins by the perfused rat liver. Acta Univ Palacki Olomuc Fac Med. 1998;141:39-40.

8. Provinciali M, Papalini F, Orlando F, Pierpaoli S, Donnini A, Morazzoni P, Riva A, Smorlesi A. Effect of the silybin-phosphatidylcholine complex (IdB 1016) on the development of mammary tumors in HER-2/ neu transgenic mice. Cancer Res. 2007;67:2022-2029.

9. Lorenz D, Lucker P, Mennicke W, Wetzelsberger N. Pharmacokinetic studies with silymarin in human serum and bile. Methods Find Exp Clin Pharmacol. 1984;6:655-661.

10. Schandalik R, Perucca E. Pharmacokinetics of silybin following oral administration of silipide in patients with extrahepatic biliary obstruction. Drugs Exp Clin Res. 1994;20:37-42.

11. Schulz H, Schurer M, Krumbiegel G, Wachter W, Weyhenmeyer R, Seidel G. The solubility and bioequivalence of silymarin preparations. Arzneimittelforschung. 1995;45:61-64. German.

12. Barzaghi N, Crema F, Gatti G, Pifferi G, Perucca E. Pharmacokinetic studies on IdB 1016, a silybin-phosphatidylcholine complex, in healthy human subjects. Eur J Drug Metab Pharmacokinet. 1990;15: 333-338.

13. Morazzoni P, Magistretti M, Giachetti C, Zanolo G. Comparative bioavailability of silipide, a new flavanolignan complex, in rats. Eur $J$ Drug Metab Pharmacokinet. 1992;17:39-44.

14. Arcari M, Brambilla A, Brandt A, et al. A new inclusion complex of silibinin and beta-cyclodextrins: in vitro dissolution kinetics and in vivo absorption in comparison with traditional formulations. Boll Chim Farm. 1992;131:205-209. Italian.

15. Chen W, Xia H, Wu W. Optimized preparation of silymarin dripping pills by a central composite design response surface method. Chin Trad Herbal Drugs. 2005;36:679-683.

16. Seo YG, Kim DH, Ramasamy T, et al. Development of docetaxelloaded solid self-nanoemulsifying drug delivery system (SNEDDS) for enhanced chemotherapeutic effect. Int J Pharm. 2013;452:412-420. 
17. Lladser M, Medrano C, Arancibia A. The use of supports in the lyophilization of oil-in-water emulsions. J Pharm Pharmacol. 1968;20: $450-455$.

18. Myers S, Shively M. Solid-state emulsions: the effects of maltodextrin on microcrystalline aging. Pharm Res. 1993;10:1389-1391.

19. Kim GG, Poudel BK, Marasini N, et al. Enhancement of oral bioavailability of fenofibrate by solid selfmicroemulsifying drug delivery systems. Drug Dev Ind Pharm. 2013;39:1431-1438.

20. Nakashima T, Shimizu M, Kukizaki M. Particle control of emulsion by membrane emulsification and its applications. Adv Drug Deliv Rev. 2000;45:47-56.

21. Oh DH, Balakrishnan P, Oh YK, Kim DD, Yong CS, Choi HG. Effect of process parameters on nanoemulsion droplet size and distribution in SPG membrane emulsification. Int $J$ Pharm 2011;404:191-197.

22. Society of Toxicology. Guiding Principles in the Use of Animals in Toxicology. Reston, VA: Society of Toxicology; 2008. Available from: http://www.toxicology.org/AI/FA/guidingprinciples.pdf. Accessed August 6, 2013.

23. Choi YK, Poudel BK, Marasini N, et al. Enhanced solubility and oral bioavailability of itraconazole by combining membrane emulsification and spray drying technique. Int J Pharm. 2012;434:264-271.

24. Pradhan R, Lee DW, Choi HG, Yong CS, Kim JO. Fabrication of a uniformly sized fenofibrate microemulsion by membrane emulsification. J Microencapsul. 2013;30:42-48.

25. Fariss MW, Bryson KF, Hylton EE, Lippman HR, Stubin CH, Zhao XG. Protection against carbon tetrachloride-induced hepatotoxicity by pre-treating rats with the hemisuccinate esters of tocopherol and cholesterol. Environ Health Perspect. 1993;101:528-536.

26. Lee SN, Poudel BK, Tran TH, et al. A novel surface-attached carvedilol solid dispersion with enhanced solubility and dissolution. Arch Pharm Res. 2013;3:79-85.

27. Marasini N, Yan YD, Poudel BK, Choi HG, Yong CS, Kim JO. Development and optimization of self-nanoemulsifying drug delivery system with enhanced bioavailability by Box-Behnken design and desirability function. J Pharm Sci. 2012;101:4584-4596.

28. Vladisavljevic GT, Williams RA. Recent developments in manufacturing emulsions and particulate products using membranes. Adv Colloid Interface Sci. 2005;113:1-20.
29. Kim HJ, Yoon KA, Hahn M, Park ES, Chi SC. Preparation and in vitro evaluation of self-microemulsifying drug delivery systems containing idebenone. Drug Dev Ind Pharm. 2000;26:523-529.

30. Tsukada Y, Hara K, Bando Y, et al. Particle size control of poly (dl-lactide-co-glycolide) nanospheres for sterile applications. Int $J$ Pharm. 2009;370:196-201.

31. Vladisavljević GT, Williams RA. Manufacture of large uniform droplets using rotating membrane emulsification. J Colloid Interface Sci. 2006;299:396-402.

32. Vladisavljević GT, Duncanson WJ, Shum HC, Weitz DA. Emulsion templating of poly(lactic acid) particles: droplet formation behavior. Langmuir. 2012;28:12948-12954.

33. Vladisavljevi G, Schubert H. Preparation of emulsions with a narrow particle size distribution using microporous $\alpha$-alumina membranes J Dispers Sci Technol. 2003;24:811-819.

34. Oh DH, Kang JH, Kim DW, Lee BJ, Kim JO, Yong CS, Choi HG. Comparison of solid self-microemulsifying drug delivery system (solid SMEDDS) prepared with hydrophilic and hydrophobic solid carrier. Int J Pharm. 2011;420:412-418.

35. Ee S, Duan X, Liew J, Nguyen Q. Droplet size and stability of nanoemulsions produced by the temperature phase inversion method. Chem Eng J. 2008;140:626-631.

36. Quan Q, Kim DW, Marasini N,et al. Physicochemical characterization and in vivo evaluation of solid self-nanoemulsifying drug delivery system for oral administration of docetaxel. J Microencapsul. 2013;30:307-314

37. Sethia S, Squillante E. Solid dispersion of carbamazepine in PVP K30 by conventional solvent evaporation and supercritical methods. Int $J$ Pharm. 2004;272:1-10.

38. Grau MJ, Kayser O, Müller RH. Nanosuspensions of poorly soluble drugs - reproducibility of small scale production. Int J Pharm. 2000;196:155-157.

39. Albers J, Alles R, Matthe, K, Knop K, Nahrup J, Kleinebudde P. Mechanism of drug release from polymethacrylate-based extrudates and milled strands prepared by hot-melt extrusion. Eur J Pharm Biopharm. 2009;71:387-394.

40. Xi J, Chang Q, Chan CK, et al. Formulation development and bioavailability evaluation of a self-nanoemulsified drug delivery system of oleanolic acid. AAPS PharmSciTech. 2009;10:172-182.
International Journal of Nanomedicine

\section{Publish your work in this journal}

The International Journal of Nanomedicine is an international, peerreviewed journal focusing on the application of nanotechnology in diagnostics, therapeutics, and drug delivery systems throughout the biomedical field. This journal is indexed on PubMed Central,

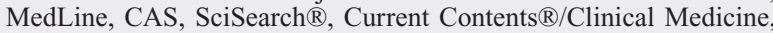

\section{Dovepress}

Journal Citation Reports/Science Edition, EMBase, Scopus and the Elsevier Bibliographic databases. The manuscript management system is completely online and includes a very quick and fair peer-review system, which is all easy to use. Visit http://www.dovepress.com/ testimonials.php to read real quotes from published authors. 